\title{
Gold Fibre Brushes
}

\section{THEIR PROMISE FOR FUTURE HIGH-TECHNOLOGY APPLICATIONS}

\section{Doris Kuhlmann-Wilsdorf}

Department of Materials Science, University of Virginia, Charlottesville, VA., U.S.A.

\begin{abstract}
A new type of electrical brush bas been developed to accommodate the otherwise unmet demands of future high technology. It consists of metal fibres finer than a buman bair protruding from a solid matrix very much like the fibres of a camel bair brush. Laboratory tests as well as theory indicate that the new brushes can by far outperform the best currently available brushes especially if the fibres are made of gold or gold alloys. Even betterperformances are expected to be reached with a second generation brush of similar type embodying yet thinner fibres.
\end{abstract}

Gold is well established as a contact material. The freedom exhibited by the metal from insulating surface films under a very wide range of conditions and its outstanding corrosion resistance, together with its easy formability coupled with ease of alloying and joining, leave gold without a serious challenger. Thus it is almost taken for granted that wherever contacts must be repeatedly made and broken using light forces, and especially where high reliability is required, the switches will be made of or be coated with gold.

Furthermore, for sliding wire contacts used in the transmission of low currents under small forces such as in guidance systems, gold or gold electrodeposits are unsurpassed for reliable operation. Even so, gold is seldom thought of as a likely material for application in heavy current technology, for example in making electrical brushes conducting hundreds of amperes. The indications are, however, that this situation will change in the future. The reason is that a number of hightechnology developments, now on the drawing board or in various stages of experimental design, require electrical brushes with performance characteristics greatly superior to those available so far. Examples are high performance homopolar motors and generators, based either on superconducting or ordinary magnets, energy storage and conversion devices, for example those which would be needed for pulsed fusion energy, and rail launchers or 'mass throwers' $(1,2)$.

Perhaps the most promising new type of brush proposed to solve this technological bottleneck is the metal fibre brush. This is quite literally a brush, but made of metal fibres rather than bristles, these being often much thinner than a human hair. Figure 1 shows an example. What ordinarily serves as a 'brush', transmitting current across an interface between parts of a circuit in relative motion, is almost always a 'monolithic' (that is one compact piece) metal-graphite brush the components of which are compacted with a binder material.

Much research will still have to be carried out to develop and perfect metal fibre brushes that will reliably satisfy the various requirements of future technology which cannot be met by the present monolithic brushes. Specifically, these requirements include the ability to operate at very high speeds and current densities with low power losses. Thus far metal fibre brushes, and the related metal wire brushes, have been made only in the laboratory or for pilot machinery (3-7). This article presents the argument for the viewpoint that metal fibre brushes are the best possible solid brushes, in principle surpassing in their performance not only monolithic brushes, but also foil (8) and metal wire $(6,7)$ brushes. Theoretically, brushes of liquid metal might possess superior properties. However, because of design and maintenance complications, such brushes are not likely to be competitive when their metal fibre counterparts with the anticipated properties become commercially available. Finally, it is very probable that a high fraction of future fibre brushes will use gold as the fibre material because of its superb surface characteristics.

\section{Why Metal Fibre Brushes?}

It has been stated above that, in principle, metal fibre brushes are likely to be the best possible heavy current conductors. In presenting the case for this point of view it is necessary to begin by examining the origin of the power losses when electrical brushes transmit currents across moving interfaces.

For high-technology applications, 'the best possible brush' means that brush which, with an acceptable lifetime, can conduct the highest current density at the fastest speed and/or with the lowest possible power loss. Since in sustained operation the heat developed in the brush must be transported away by means of some type of forced cooling, there is generally a strong incentive to keep it at a low level. In addition in some brush applications such as homopolar motors/generators and in energy storage devices, very stringent limits are placed on permissible losses lest the devices become uneconomical. However, even if neither of the above conditions are evident and the heat developed in the brushes is of no direct concern, it is indirectly most important when high current densities must be achieved since local melting at contact spots will typically cause brush failure. When at rest, the local temperature at the contact spot is roughly proportional to the applied voltage (9). There- 
fore, the critical local temperature tends to occur at some specific voltage' drop for brushes at rest, meaning at some specific wattage loss per ampere conducted. The frictional and joule heats are additive and, together, limit the maximum current carrying capacity of a brush.

As already indicated, the brush 'loss' under discussion comes from two sources. The first is electrical, joule heat being generated at a rate per ampere of:

$$
L_{E}=R_{B} I
$$

where $R_{B}$ is the electrical resistance of the brush and interface, and $I$ is the current. The second is mechanical, heat being produced as a result of friction. Expressing this also in terms of wattage per ampere and distegarding windage losses, the mechanical loss is given by:

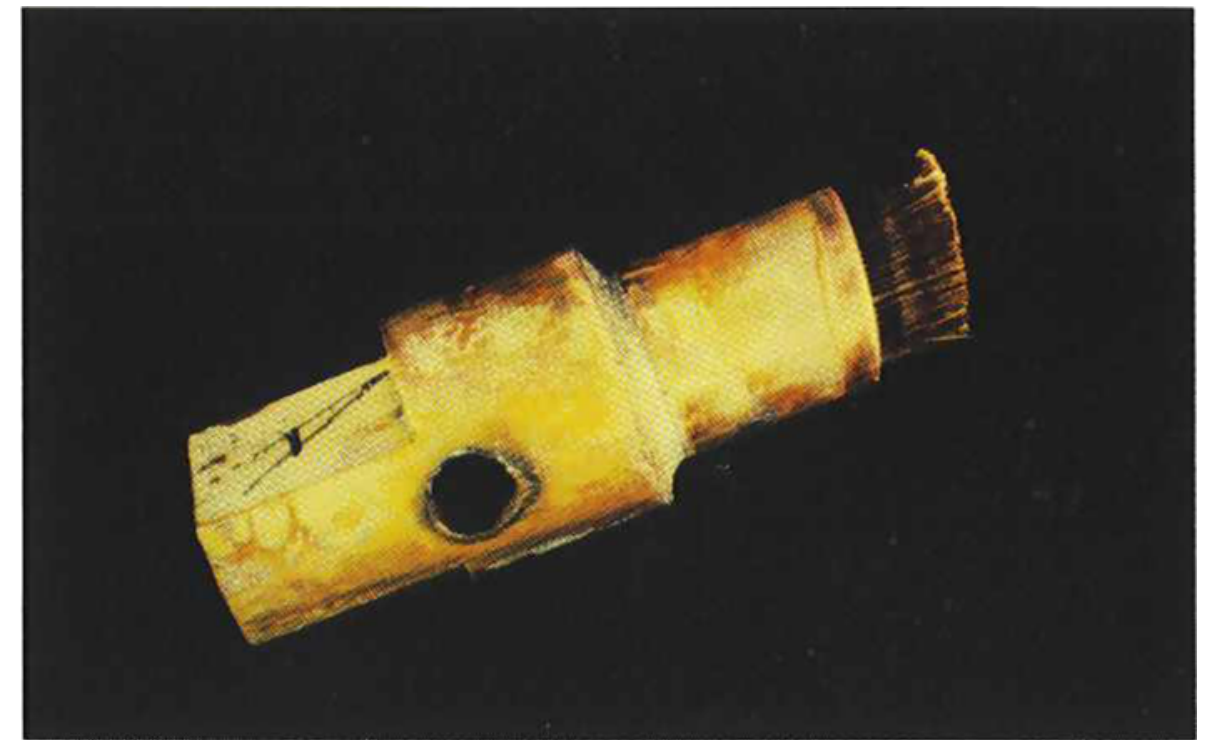

Fig. 1 A fibre brush made of gold fibres of about $0.5 \mathrm{~cm}$ length and $20 \mu \mathrm{m}$ thickness, that is about one third that of a human hair, protruding from a copper matrix. The best data so far obtained, for example those incorporated in Figs. 6 to 8, were obtained with brushes of this type

$$
L_{M}=\mu \nu P / I
$$

where $v$ is the relative velocity between the two contact surfaces, $\mu$ is the coefficient of friction, and $P$ is the 'brush force', that is the normal force with which the brush is pressed against the opposite component, be it a slip ring, a commutator, or a straight piece of metal (as in a mass thrower).

The electrical resistance of a brush, $R_{B}$, decreases with brush force, $P$. This is because $A_{b}$, the actual area of mechanical loadbearing contact between the brush and the opposite member, indeed between any two solids, is a unique function of $P$ and is independent of the apparent area of contact, that is, in our case, the sliding area of the brush, $A_{B}$. In fact, for brushes, $A_{b} \ll A_{B}$ always, and the area of $A_{b}$ is composed of individual contact spots which, to the extent that current passes through them, are called 'a-spots'. Consequently, if current passes through $A_{b}$ and cannot cross the intervening gaps between the a-spots then, at the interface, the brush acts as if it consisted of a number (say $n$ ) of isolated conducting areas which are electrically 'in parallel'. The dependence of $R_{B}$ on $P$ therefore arises because by increasing $P$ the area of $\dot{A}_{b}$ is enlarged, of course, and hence $R_{B}$ is decreased. Figure $2 a$ qualitatively indicates the resultant dependence of the total brush 'loss'

$$
L_{T}=L_{M}+L_{E}
$$

on the brush force $P$, assuming that the conduction is exclusively ohmic and the coefficient of friction is constant, while Figure $2 \mathrm{~b}$ similarly depicts $L_{T}$ as a function of current, $I$, all other parameters being held constant in each case.

It can be shown that when $L_{M}$ and $L_{E}$ are of the form $\gamma P^{\beta}$ and $C / P^{b}$ respectively, $L_{T}$ is minimized when $L_{E}=(\beta / b) L_{M}$ : that is $L_{E}=L_{M}$ for $b=\beta$, as is often approximately true. Quite commonly, $L_{E}$ is roughly inversely proportional to $P$ while $L_{M}$ is proportional thereto, as depicted in Figure 2. As a result $L_{T}$ is typically minimized when $L_{T} \simeq L_{M}$, which may be teadily shown by differentiation of the function $C / P+\gamma P$. For a more detailed determination of the dependence of $R_{B}$ on $P$ and other parameters it is important to realize that $R_{B}$ is the sum of three components, as follows:

$$
R_{B}=R_{\Omega}+R_{F}+R_{C}
$$

Here $R_{\Omega}$ is the ohmic resistance of the brush body. $R_{F}$ is the 'film resistance', which is due to the ubiquitous presence of surface films on all metals in the atmosphere, whether they be visible or consist only of a monomolecular layer of adsorbed gas. Finally, $R_{C}$ is the so-called constriction resistance.

Little discussion is needed regarding $R_{\Omega}$ which is largely self explanatory. If, say, the brush is monolithic of length $l_{B}$, the brush material has a resistivity $\rho_{B}$, and if the current is fed in at the end furthest from the interface, the ohmic resistance is simply $R_{\Omega}=\rho_{B} l_{B} / A_{B}$. For more complicated geometries $R_{\Omega}$ may have to be determined experimentally.

The film resistance is equally simple to treat, assuming that the film is uniform everywhere. This assumption is justified in the case of high-performance brushes, as is also the assumption 


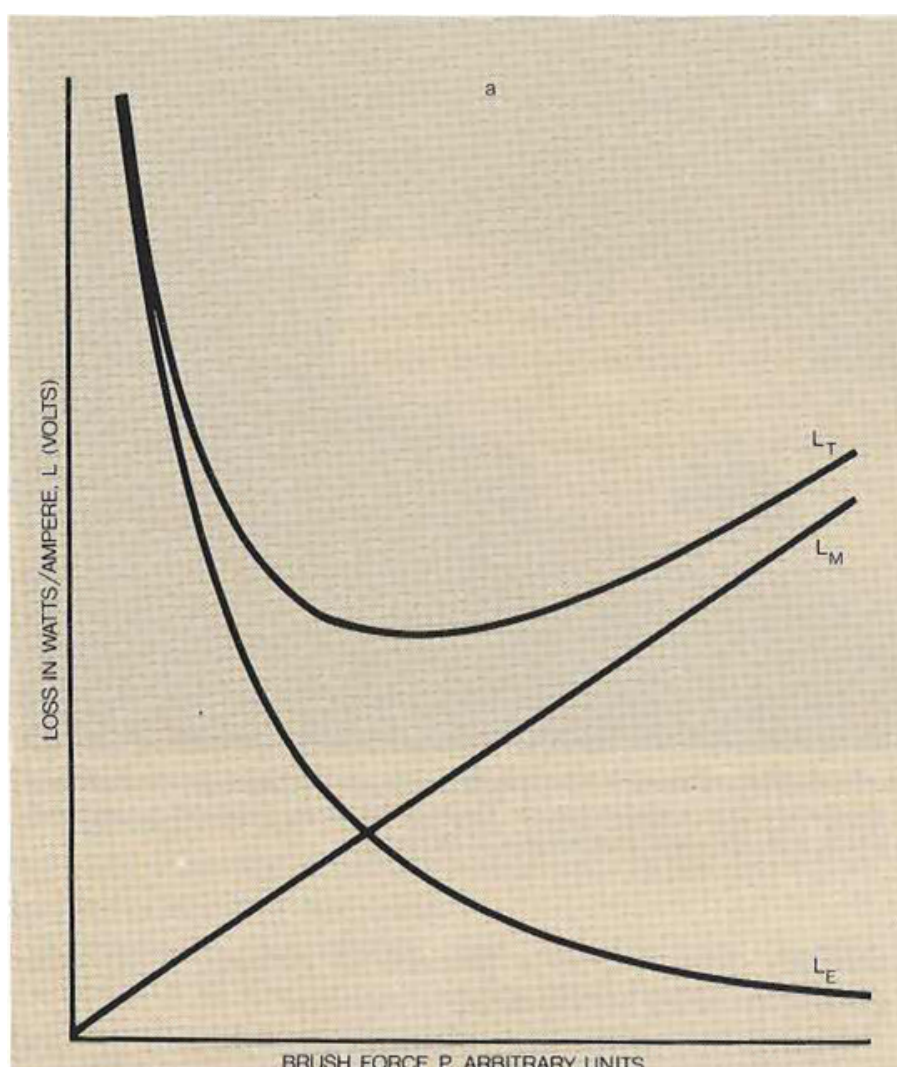

BRUSH FORCE P, ARBITRARY UNITS

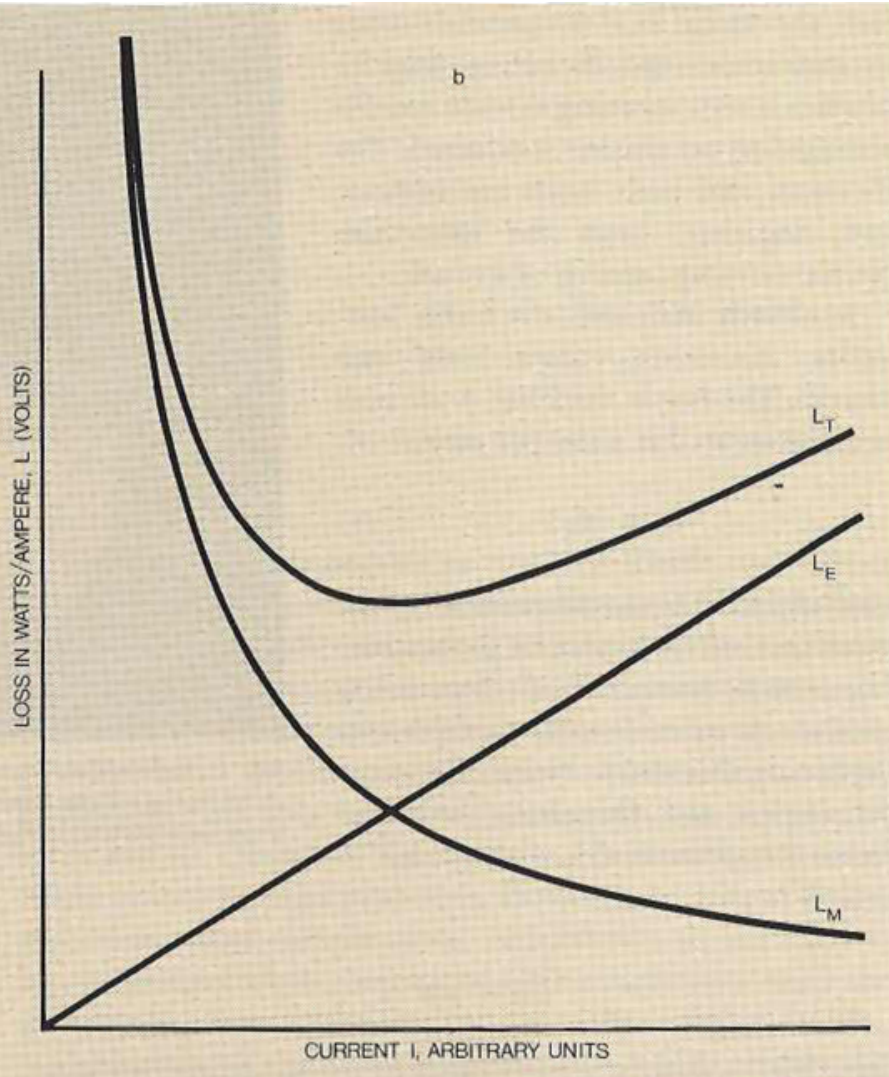

Fig. 2 The dependence of the rate of heat developed per ampere conducted through a brush as a function of applied force (Fig. 2a) and current (Fig. 2b) assuming that all other parameters are constant. The power loss is due to two causes, electrical, indicated by the symbol $L_{E}$ and frictional heat, symbol $L_{M}$, giving a total loss $L_{T}$, all measurable in watts/ampere, that is volts

that the area of the a-spots equals $A_{b}$. In this case the current passing through the area $A_{b}$ must, at the interface, also cross a film of equal area. Therefore

$$
R_{F}=\sigma_{F} / A_{b}
$$

where $\sigma_{\mathrm{F}}$ is the 'film resistivity' (normally measured in ohm $\mathrm{m}^{2}$ ), that is the reciprocal of the electrical conductivity per unit area of the surface film.

Lastly, the constriction resistance is due to the crowding of the current flow lines as they pass through the a-spots. If the a-spots are assumed to be circular and of similar sizes, their average radius $a$ is, for $\mathrm{n}$ a-spots, found to be:

$$
a=\left(A_{b} / \pi \mathrm{n}\right)^{1 / 2}
$$

Asshown by R. Holm (9) the constriction resistance per a-spot is, to a first approximation, comparable to that of a cube of edge length $2 a$ straddling the interface. Thus, if the resistivity of the brush material is $\rho_{B}$ and that of the conductor actoss the interface is $\rho_{R}$, the constriction resistance is:

$$
R \simeq \bar{\rho} / 2 a \simeq \bar{\rho}\left(\mathrm{n} / A_{b}\right)^{1 / 2}
$$

where $\bar{\rho}=\left(\rho_{B}+\rho_{R}\right) / 2$. The total constriction resistance for the $\mathrm{n}$ parallel contact spots is thus:

$$
R_{C} \simeq \bar{\rho} /\left(\mathrm{n} A_{b}\right)^{1 / 2}
$$

In order to fully understand the dependence of $R_{B}$, and thus $L_{T}$, on the brush force, it is necessary to consider the role of $A_{b}$. This, in fact, depends on the state of the load-bearing spots at the interface. To a first approximation only two limiting cases need to be considered. The first is that the contact spots are fully plastic, hardened to the saturation hardness $H$ of the softer of the two materials meeting at the interface (which generally is the brush). In that condition:

$$
{ }_{p} A_{b}=P / H
$$

where the subscript $p$ is meant to remind the reader that we are concerned here with plastically deformed a-spots.

Gold Bull., 1983, 16, (1) 
Alternatively, if the load is light and the number of a-spots very large, they may be elastically stressed. Clearly, in that case the average normal pressure at the a-spots is well below $H$, and correspondingly, for the same load and other parameters, the value of $A_{b}$ is increased over that obtained under plastic conditions. This problem was first considered mathematically by Hertz (10) who modelled the contact spots as ellipsoidal asperities on one side meeting a planar surface on the other side. Using the solution of Hertz (10) as worked out for the case of spherical asperities by Huber (11), we obtain (3, 4, 5 and 9):

$$
{ }_{e} A_{b} \simeq 1.2 \pi\left(P^{2} n r_{c}^{2} / E^{2}\right)^{1 / s}
$$

Here $r_{c}$ is the local radius of surface curvature at the average a-spot and $E$ is an averaged value of Young's modulus of brush and substrate material.

In summary then, the loss in the case of a high-performance electrical brush may be written as:

$$
L_{T} \simeq \mu P v / I+\left[R_{\Omega}+\sigma_{F} l_{j} A_{b}+\bar{\rho} /\left(\mathbf{n}_{j} A_{b}\right)^{1 / 2}\right] I
$$

where ${ }_{j} A_{b}$ stands for ${ }_{p} A_{b}$ according to equation 9, or for ${ }_{e} A_{b}$ according to equation 10 when the contact spots are plastically or elastically deformed, respectively.

Considering equation 11 with the aim of reducing $L_{T}$ to its lowest possible value, and comparing monolithic metal/ graphite brushes with metal fibre brushes for use at high current densities, the superiority of the latter becomes obvious. Admittedly $\mu$ will typically be larger for metal fibre than for metal/graphite brushes, perhaps by a factor of two or three, but, for fixed $P, L_{M}$ becomes small for large currents, being inversely proportional to $I$, in which case $L_{E}$, being proportional to $I$, dominates brush performance. Correspondingly, at high current densities the penalty for increasing $\mu$ in metal fibre brushes, and thus $L_{M}$, for fixed $P$, is more than compensated for by the following decreases in $R_{B}$ as discussed below.

First, with a metal body, metal fibre brushes have a negligible value of $R_{\Omega}$ whereas in the case of monolithic metal/graphite brushes $R_{0}$ makes a significant contribution to $R_{B}$. Secondly, unlike brushes of metal/graphite, metal fibre brushes do not develop a lubrication layer. Therefore, if the brush is well constructed and properly applied, the film resistivity, $\sigma_{F}$, can attain the lowest possible value compatible with smooth sliding (as opposed to galling). This value is of the order of $\sigma_{\mathrm{F}} \simeq 1 \mathrm{x}$ $10^{-12} \mathrm{ohm} \mathrm{m^{2 }}$ and can be as low as $5 \times 10^{-13} \mathrm{ohm} \mathrm{m^{2 }}$ for gold $(4,5)$. This is much lower than the film resistivity of the ordinary metal/graphite lubrication layer of monolithic brushes $(12,13)$, Thirdly, monolithic brushes have only a relatively small number of a-spots, of the order of ten (13) and perhaps up to forty (14). Correspondingly, their constriction resistance is significant, accounting for perhaps one third of the total resistance. By contrast, for metal fibre brushes the number of contact spots is comparable with the number of fibres or larger $(4,5)$ so that $R_{C}$ is negligible in accordance with equation 8 .

Thus bulk-, film- and constriction resistances are all drastically reduced in metal fibre as compared with metal/ graphite brushes.

\section{Some Mechanical Aspects of Brush Performance}

The principles discussed above, although independently developed in our laboratory, have also been pursued by other researchers. The reasons why metal fibre brushes have not been developed previously for high-performance purposes are mainly historical. The first 'brushes' were presumably the real wire brushes from which the name derived and which can be recalled as being used in Whimshurst machines and similar electrostatic generators. Such wire brushes performed well in these because of the low currents and high voltages employed, these being such that, in fact, current was conducted across the gap mainly by corona discharge rather than by electrons through a-spots. When higher current densities and lower voltages were required, wire brushes were not satisfactory. They tended to scratch the opposing surface, had a high resistance, and would give rise to sporadic arcing when the wires intermittently bounced off the other surface. Indeed, bouncing of brushes, with the attendant highly unwelcome arcing which causes damage to brush and rotor besides leading to strong electrical noise, is a problem also encountered with monolithic brushes, a point to which we shall return presently.

Once the self-lubricating graphite and metal/graphite brushes had been developed, they very quickly displaced the old-style wire brushes almost completely. Specifically, lubrication, by which the mechanical loss is reduced and the brush is made to run smoothly without exhibiting stick slip, was recognized as so beneficial that the remaining minority of metal/metal contact brushes or similar devices, for example elastic clamps on axles, were also lubricated, as indeed are many switches in order to allow them to work more smoothly and without welding at the contact points. Unlubricated brushes were thereafter widely considered to be unsatisfactory in principle.

That fibre brushes are mechanically compliant, thus permitting them to run at low loads, and that they effectively eliminate the constriction resistance were recognised as most attractive features and, with the recognition that the performance of electric brushes was improved by lubrication, the invention of the metal plated carbon fibre brush followed (14). However, the bulk resistance of this type of brush is still fairly high and, to the extent that a graphite-rich lubrication layer is deposited, the film resistance is also comparatively large.

Last but not least among the obstacles to development of metal fibre brushes, the production of fibres of the requisite 


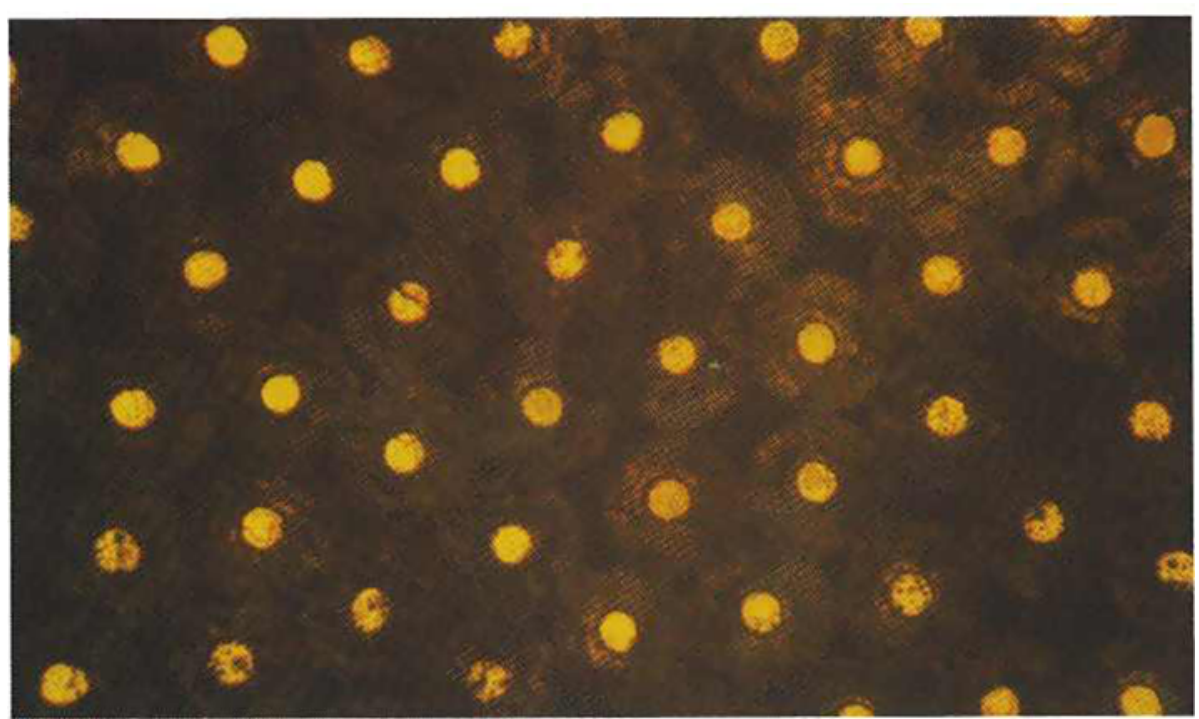

Fig. 3 Micrograph of the cross section of 'brush stock' at an early stage in the production process. At this point the gold rod or wire, encased in copper tubing, has been drawn down to an outer diameter of about $0.25 \mathrm{~mm}$, was cut into pieces and rebundled into another copper tube, not visible in this picture. Gaps between the individual strands arè still clearly visible

brushes. It has already been mentioned that monolithic brushes tend to 'bounce', causing arcing. This problem is reduced or inhibited by increasing the brush force $P$. In practical terms the required brush pressures to prevent intermittent arcing are rarely less than $30000 \mathrm{~N} / \mathrm{m}^{2}$, and more typically are

small diameters was initially considered to be quite difficult. At the University of Virginia, without any prior research experience with electrical brushes but with a materials science background instead, the latter problem appeared to be manageable. Furthermore, from the materials science viewpoint it seemed that lubrication would be unnecessary when the load on each contact spot was made so small (for example a load below $10 \mathrm{mgf}$ perfibre) that almost no dislocation motion would be triggered. Such low forces would be attained, for example, with fibre diameters of, say, $d \leq 40 \mu \mathrm{m}$ at brush pressures $p_{B}=P / A_{B}$ $\simeq 4000 \mathrm{~N} / \mathrm{m}^{2}$ and 'packing fractions' $f \geq 0.05$ (the latter meaning the fraction of the interface $A_{B}$ which is occupied by fibres as compared to the space left between them to permit free flexing of individual fibres).

This, then, brings us again to the purely mechanical aspects of

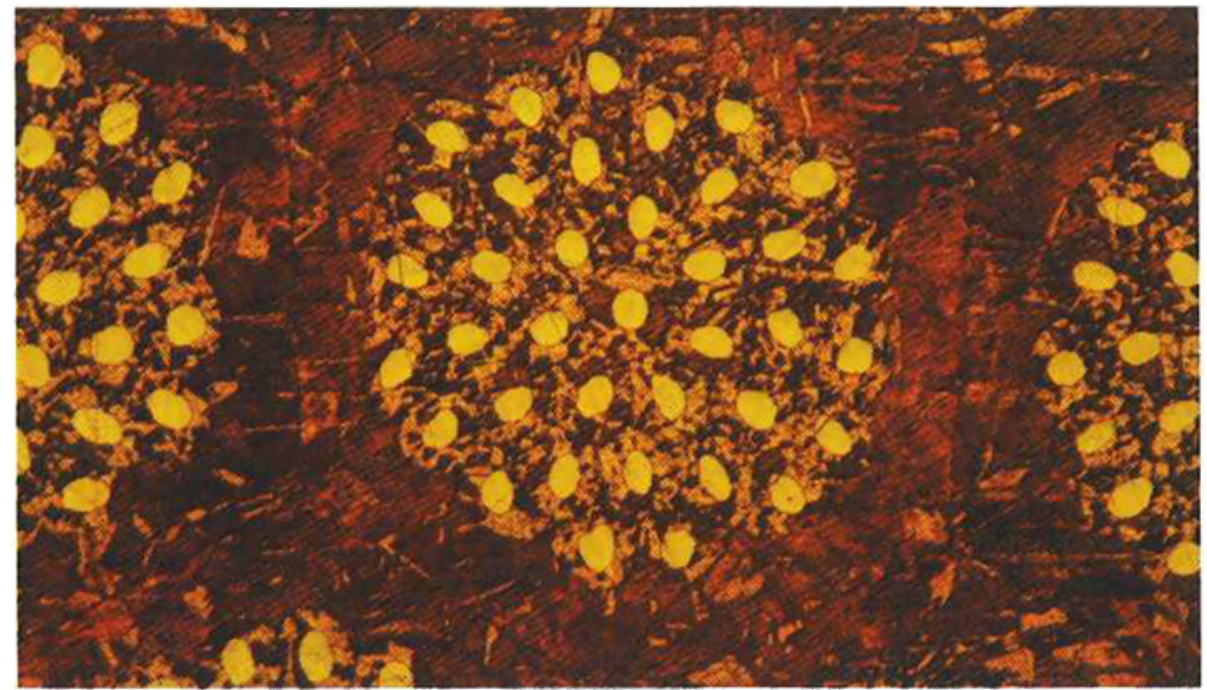

twice this figure, increasing with speed. Therefore it is very often impossible to adjust $P$ to the minimum loss of Figure 2a, particularly at high speed. In fact, monolithic brushes are commonly run at some fixed pressure, in the region of $p_{B}=$ $60000 \mathrm{~N} / \mathrm{m}^{2}$, regardless of current, to avoid arcing.

The reason for the required large forces is, of course, the rigidity of the brushes, in combination with their inertia. Fibre brushes, however, can be made as soft as velvet, and therefore can be used at very much smaller pressures, for example 4000 $\mathrm{N} / \mathrm{m}^{2}$ (see the example given above) even at high speeds. The mechanical loss $L_{M}$ is correspondingly greatly reduced.

\section{Making and Testing Metal Fibre Brushes}

Beginning with a rod of the intended fibre material inserted into a tubing of the matrix material, with the dimensions adjusted for the planned value of the packing fraction, fibre brushes have been made by drawing through dies until the desired fibre diameter is achieved. Commonly this requires intermediate rebundling into matrix fibre tubing which then is again drawn

Fig. 4 As Fig. 3 but taken at a later stage, after the outer copper tubing surrounding the bundle shown in Fig. 3 was drawn down to about $0.25 \mathrm{~mm}$, rebundled into yet another copper tube and 'drawn to closure', meaning until no gaps remained in the brush stock. With the gold fibres now about $20 \mu \mathrm{m}$ thick, the brush stock is ready for sectioning, shaping and etching, to make a brush as shown in Fig. 1

Gold Bull., 1983, 16, (1) 
down (3). Once the final fibre thickness is attained the multifilamentary wires so produced are packed into a casing the inner dimension of which has a shape and size such that on further light drawing or rolling the desired brush size results. The purpose of this final drawing or rolling is to eliminate undue gaps between the multi-filaments in the 'brush stock' which is in the form of a rod with the desired cross-sectional area.

Figures 3 and 4 show examples of the structure of brush stock. The final brush, as in Figure 1, is obtained by first shaping one end of an appropriate length to the rotor against which the brush shall be tested, and then etching out the matrix material to the proper depth. Some of the concepts and methods involved have been further explained elsewhere (15).

The brushes were tested against a smooth rotor made of either polished or carbonized copper (16), as shown in Figure 5, using specially designed apparatus $(17,18)$. In this manner, brushes with fibre diametres between several micrometres and one tenth of a millimetre were made and tested, having various packing fractions, and fibre lengths of one to several millimetres. Fibre materials included gold, silver, palladium, platinum, copper, niobium and aluminium. With the exception of niobium all brushes could be operated satisfactorily in protective atmospheres (mainly argon or carbon dioxide, dry or humidified). Figures $6-8$ give examples of the results obtained.

Not unexpectedly, gold fibre brushes were found to give superior performances to all others, and gold was also the only fibre material which performed well in air. However, rather surprising was the fact that the film resistivities determined for all brushes properly performing in protective atmospheres were about $10^{-12} \mathrm{ohm} \mathrm{m} \mathrm{m}^{2}$. This is indicative of the presence of 'tunnelling films', that is adsorption films so thin that the electrons tunnel through them as indicated by strictly ohmic behaviour. Presumably these films consist of only one monolayer of molecules adsorbed from the atmosphere on each side.

The considerable superiority of good fibre brushes over the hitherto best commercially available brushes is seen mostclearly in Figure 8 Not only the much lower voltage drops for a given current but also, of great importance, the virtual absence of noise should be noted.

\section{Towards Quantum Mechanical Tunnelling Brushes}

The quantitative evaluation of the various measurements of brush resistance as functions of brush force and size, of speed, packing fraction, current density and fibre diameter, plus observations on the effects of ambient atmosphere, running time and surface characteristics of the mating surface for the brushes were vigorously pursued $(4,5)$. The conclusions reached included the following:

(1) There are one to three a-spots per fibre, depending somewhat on load and speed (5)

(2) The contact spots are elastically stressed

(3) The film resistivity for all metals other than gold rises significantly, and in some cases greatly, if oxygen is admitted into an initially oxygen-free atmosphere

(4) The radius of surface curvature, $r_{c}$, is similar to the fibre radius (not surprisingly considering that the fibres slide along the surface in a tilted position, so that their cylindrical surfaces touch the rotor).

These results were much as expected. However, the brush resistance fell below the theoretically computed value when fibre sizes were quite small, increasingly so as the fibres got thinner. This effect was interpreted as due to tunnelling through the annular zone about the load-bearing contact spots. Figure 9 illustrates the origin of the discussed effect somewhat schematically, using the example of a spherical asperity meeting a planar surface at a heavy and a light load. Certainly this geometry is greatly simplified, but it makes clear the salient point, namely that the annular zone around the contact spot with a gap width below some specific value, $h$, increases when the size of the load-bearing area decreases.

Following the example of Holm (9) and using the simple approximate geometry of Figure 9 (which among other simplifications neglects the effect of adhesive forces that tend to close the gap, (19)), one arrives at the following expression for the specific brush resistance $(4,5)$ :

$$
R_{B} A_{B}=\left(\sigma_{F} / K^{2}\right)\left[E^{2} d^{2} / p_{B}^{2} r_{c}^{2} 70 f\right]^{1 / 3}
$$

with

$$
K^{2}=1+2\left(r_{c} s^{3} / d^{4}\right)^{1 / s}\left(f E / p_{B}\right)^{2 / s}
$$

where $K^{2}-1$ is the ratio of conduction by tunnelling through the annular gaps about the a-spots, to the total conduction. In equation $12, p_{B}$ stands for $P / A_{B}$ and one a-spot per fibre is assumed, while all other symbols have the same meaning as before, including $f$ the packing fraction (rarely more than 0.2 ). Finally, $s$ is a cut-off distance. Mathematically it stands for the gap width (corresponding to $b$ in Figure 9) up to which, in the geometry of Figure 9, tunnelling may be assumed to take place at no extra resistivity above $\sigma_{F}$, and beyond which current conduction is neglected. In fact, as indicated before, the geometry in Figure 9 is greatly simplified, and, strictly, adhesive forces should not be neglected. However, $s$ can be regarded as a useful parameter which may be determined from the experimental data. It was consequently found to be approximately $0.5 \mathrm{~nm}(4,5)$.

All this might be of only academic interest, except that according to equation $12, K^{2}$ can easily become large compared to unity, in which case the conduction takes place over a much larger area than $A_{b}$, namely by quantum mechanical tunnelling 


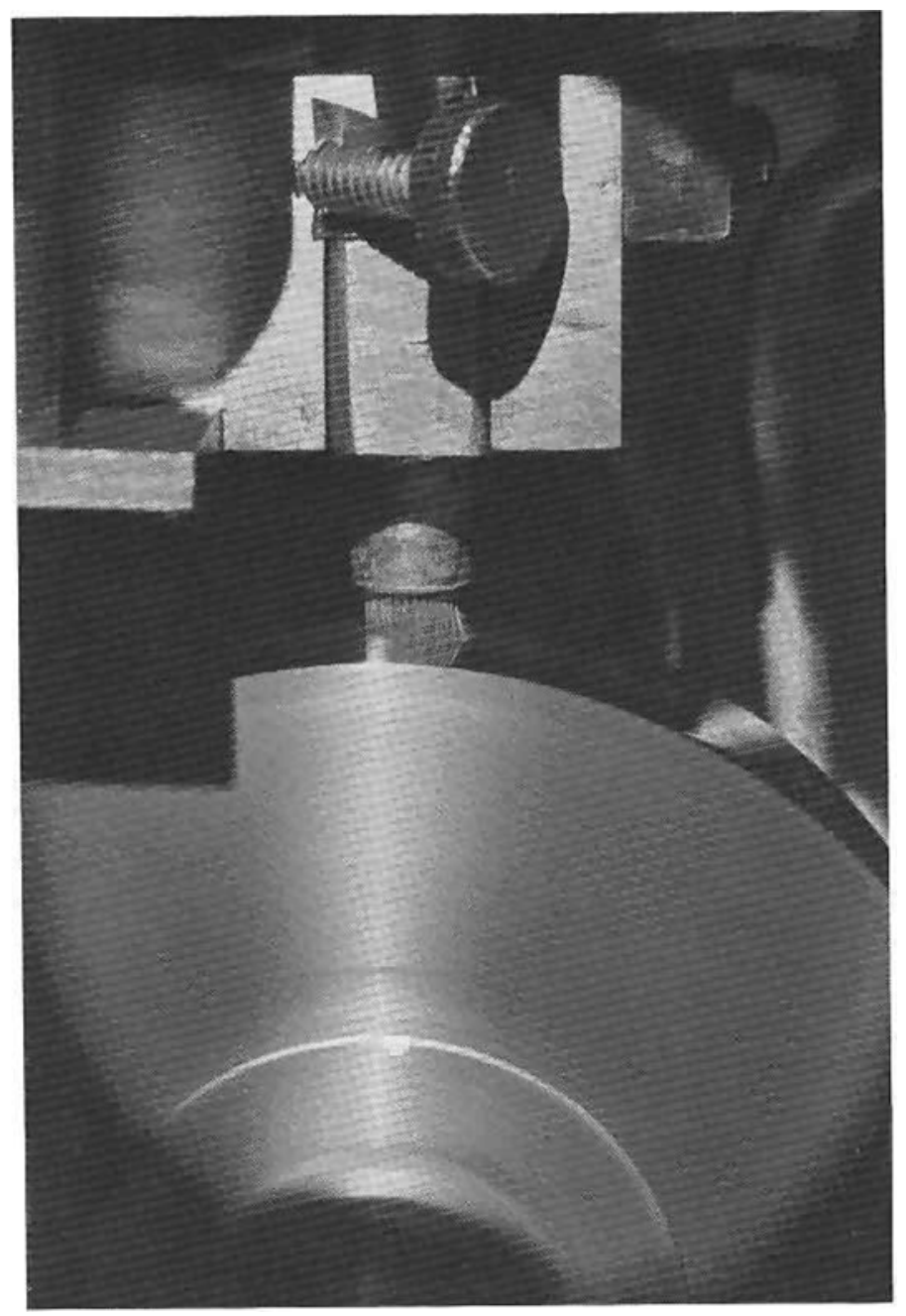

Fig. 5 A silver fibre brush of similar dimensions to the brush shown in Fig. 1 during testing against a polished copper rotor of diameter $8.25 \mathrm{~cm}$. The brush holder is obscured by parts of the device used for monitoring brush wear and deformation. Note the mirror image of the fibres reflected from the rotor surface

through the annular regions indicated in Figure 9. Brushes which conduct in this manner have been dubbed $Q M$ brushes.

For $r_{c}=d / 2$ as previously indicated, equation 12 reduces to:

$$
R_{B} A_{B} \simeq 0.25 \sigma_{F} d / s f
$$

Numerical calculations show that the specific brush resistance predicted by equation 13 can be extraordinarily low for values of the parameters which appear to be in reach. For example, at $\sigma_{F}=1 \times 10^{-12} \mathrm{ohm} \mathrm{m}{ }^{2}$ with $s=0.5 \mathrm{~nm}$ according to the previous experimental results $(4,5)$ :

$$
R_{B} A_{B} \simeq 5 \times 10^{-4}\left(\mathrm{ohm} \mathrm{m}^{2}\right) d / f
$$

This implies that for, say, fibres of thickness $d=1 \mu \mathrm{m}$ at a packing fraction $f=0.1$, a brush of area $A_{B}=1 \mathrm{~cm}^{2}$ would have a resistance of $R_{B}=0.05$ mohm, almost independent of the applied brush force. If so, a current of $5000 \mathrm{~A}$ could be passed through $1 \mathrm{~cm}^{2}$ of such a brush material at an applied voltage of $0.25 \mathrm{~V}$. This is a much larger current density than macroscopic circuits can support in continuous operation.

The theory embodied in equations $12-14$ has as yet not been proven conclusively. However, all measurements to date are compatible with it and there is no doubt that brushes with fine fibres have considerably lower resistance than that computed from equation 12 with $K=1$. Nevertheless, experimental scatter has been too large and fibre diameters have not been sufficiently thin to establish quantitative compliance with the theory. In fact, $Q M$ brushes for which $K \gg 1$ are still very difficult to produce although their production should not pose a problem once the developmental work is completed. A $Q M$ brush with gold fibres of modest specifications, that is $d \simeq 2 \mu \mathrm{m}$ and $f \approx 0.04$, is at present under construction, with most of the previous problems now having been solved.

Elsewhere (20), the theory has been presented in greater detail together with numerous ideas of how such brushes could be made and applied. If the latter live up to their promise the present problems associated with brushes will disappear as bottlenecks in all foreseeable high-technology applications. In these it seems very probable that brushes will be used which are made from gold or gold alloy fibres.

\section{Cost of Gold Fibre Brushes}

Thus far, all metal fibre brushes have been made with much effort in the laboratory. However, once the procedures for different metal combinations, relative sizes, and intermediate annealing treatments have been well established, the brush stock can be made in large quantities using the same methods and machinery as those developed and employed for the superconducting multifilamentary materials which are even now made on a commercial scale for superconducting magnets. Since only small lengths of brush stock are used in each brush, the cost of the brush fibre apart from the precious metal content, will thus be quite modest.

As to the gold metal content, this will amount to only about 5 to 10 per cent of the brush volume. This may be compared with a value of almost 50 per cent of silver content in the widely used silver-graphite brushes which, at this point, are the best commercially available brushes for high-technology applications.

The silver-graphite brushes wear with use, whereas gold fibre-brushes of the future should wear almost not at all. Consequently, the length of brush stock for gold fibre brushes needed per brush may be estimated at only about 20 per cent of that required for silver-graphite brushes. Therefore, for brushes of the same cross-section $A_{B}$, the gold to silver content would be 

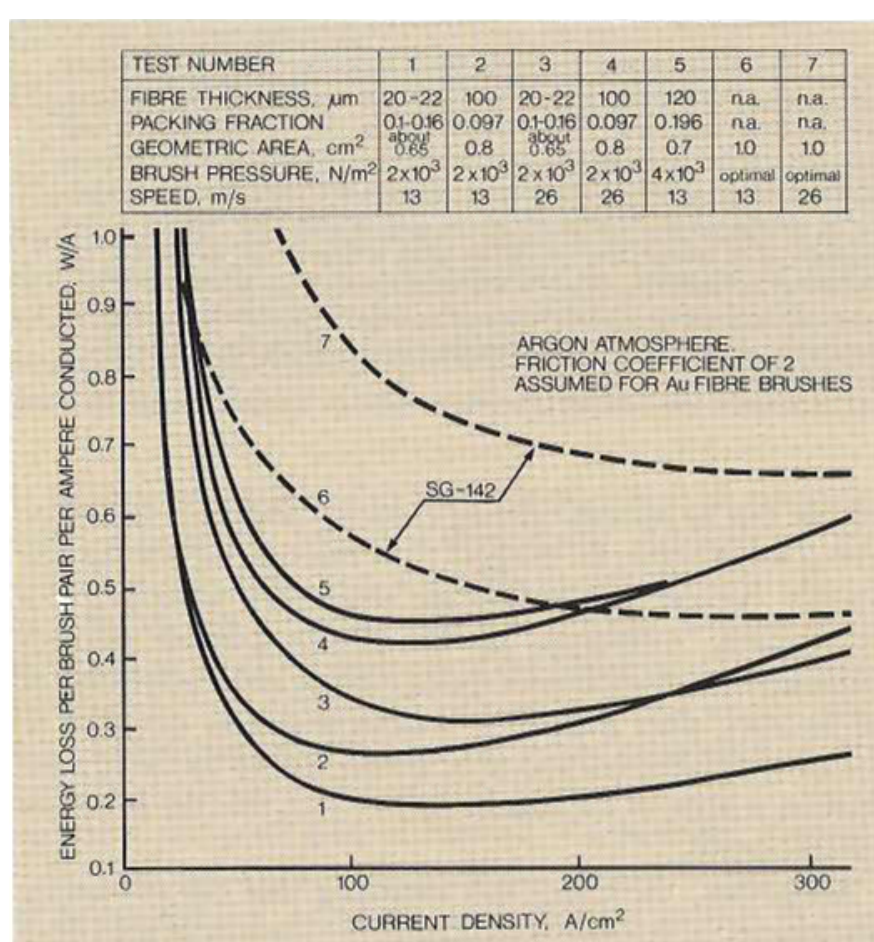

Fig. 6 Approximate values for the total power loss, $L_{T}$, for various different brushes, brush forces and speeds, as indicated, as a function of current density. Since the coefficient of friction for the fibre brushes was not measured in all cases it was estimated at 2 throughout, which is an overestimate. In fact it was nearer 0.4 and thus the brush performance was even better than is apparent from this figure. By contrast, the data obtained from the silver-graphite brushes (SG-142 with 75 weight per cent of silver, being the best commercially available brush material for high current densities) are optimal

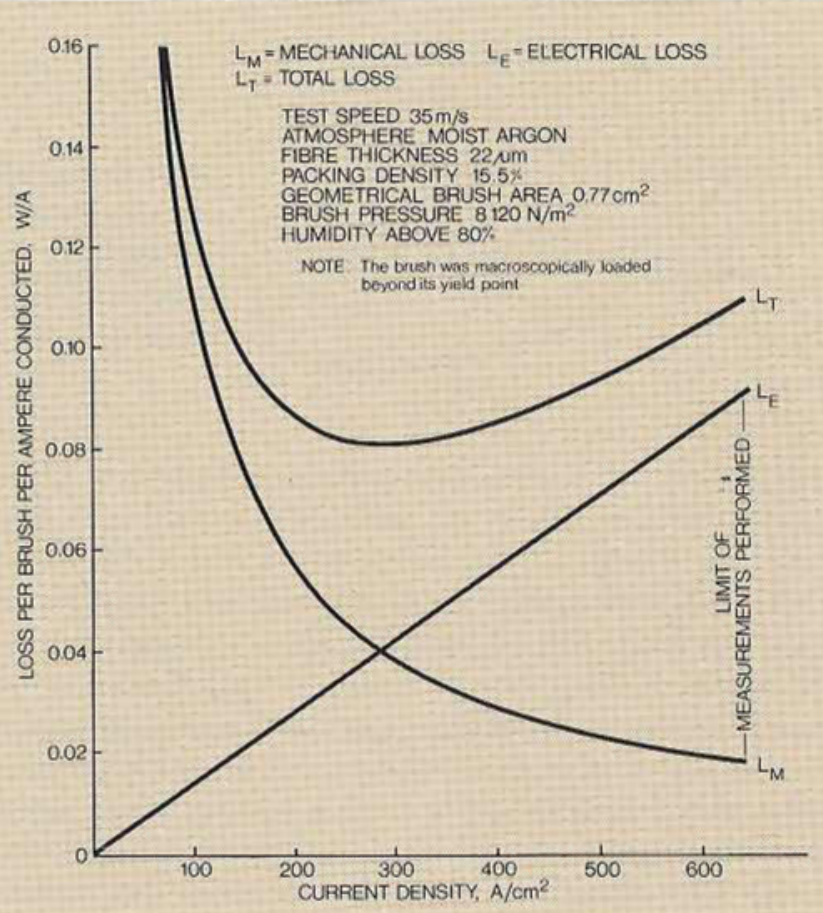

Fig. 7 Measured loss for brush 1 of Fig. 6 , but at $p_{B}=8.12 \times 10^{3} \mathrm{~N} / \mathrm{m}^{2}$, which caused plastic deformation of the brush fibres. These data represent about the best performance yet obtained, except that in later measurements, using a larger rotor, the speed could be increased to 72 $\mathrm{m} / \mathrm{s}$ resulting in a minor increase of $R_{B}$ and decrease of $\mu$. The greatly expanded scale for loss, and compressed scale for current density as compared to Fig. 6 should be noted bearing in mind, however, that in this figure the losses refer to only one brush rather than a brush pair
Fig. 8 Oscillograms for a silver-graphite brush pair (SG-142, 75 weight per cent silver) at $p_{B}=10^{5}$ $\mathrm{N} / \mathrm{m}^{2}$ and a gold fibre brush pair (identical to that in Fig. 7) at $p_{B}=9 \times 10^{3} \mathrm{~N} / \mathrm{m}^{2}$. All brushes had an area of about $0.8 \mathrm{~cm}^{2}$ and carried a cutrent density of $3.1 \times 10^{6} \mathrm{~A} / \mathrm{m}^{2}$ at a speed of $35 \mathrm{~m} / \mathrm{s}$. The brush force for the silver-graphite brushes is optimal for the given current density. The very much larger electrical loss and noise of the silver-graphite as compared to the gold fibre brushes should be noted. Furthermore the mechanical loss of the silver-graphite brushes is much larger than that shown by the gold fibre brushes, namely by a factor of between fourand five
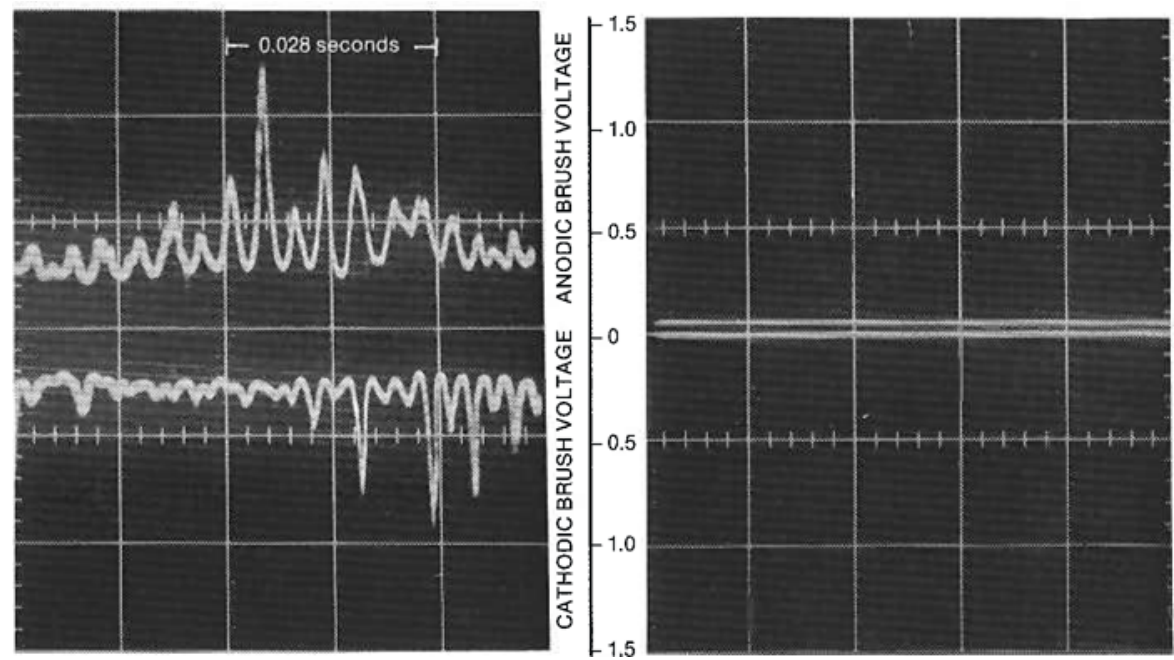

Gold Bull., 1983, 16, (1) 


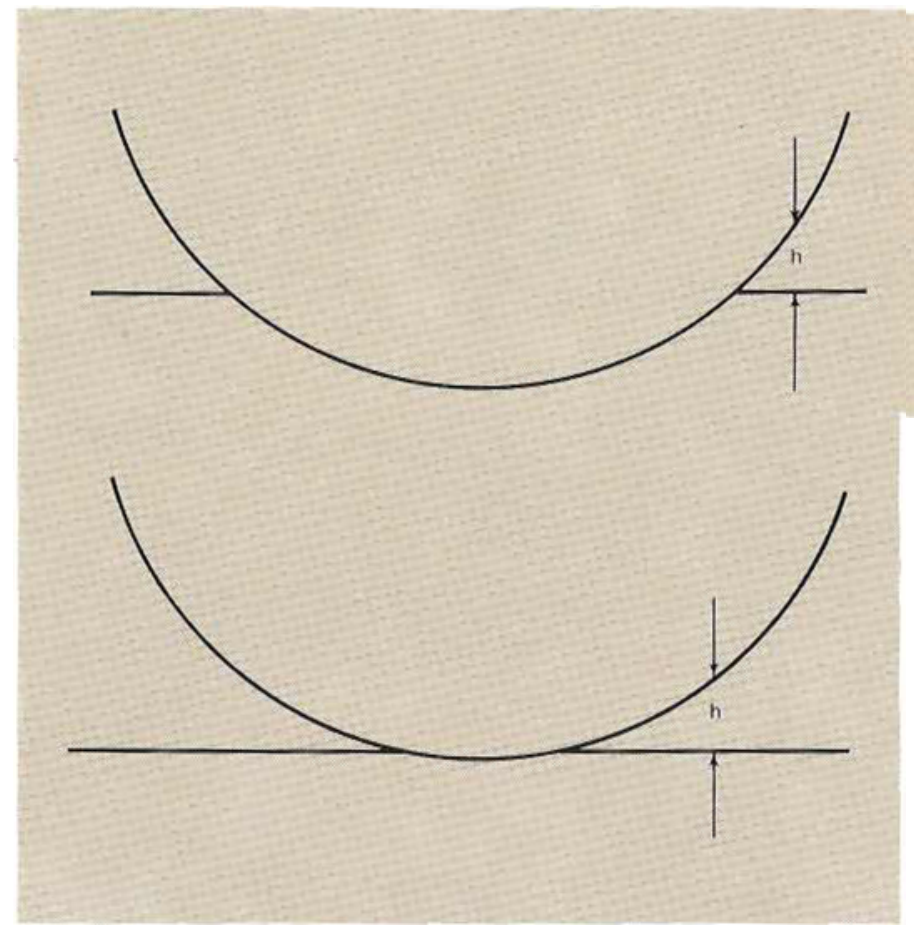

in the ratio of, perhaps, less than $0.03: 1$ by volume, or about 5 per cent by weight.

With the relative cost of silver to gold being of the order of 1 to 50 , the gold content of gold-fibre brushes would thus cost about 2.5 times that of the silver content in silver-graphite brushes of the same cross-section. However, brushes are not bought by
Fig. 9 Simplified contact geometry which explains why, with decreasing size of a-spots, the relative area through which tunnelling about the a-spots takes place is increased. The condition at the top of the figure would represent a rather large, heavily deformed a-spot, while that at the bottom reflects a small, lightly loaded a-spot. As seen the annular area with gap widths smaller than $b$ increases as the size of the load-bearing a-spot decreases. The comparable transition using a fibre brush takes place by increasing the number of fibres, and hence a-spots, at given brush pressure. In actual fact the surface contours are undoubtedly rather different. Specifically, adhesive forces will partly close the gap where the wedge angle is very small (19)

volume but by current-carrying capacity. Since this should be several times higher for the gold fibre brushes, these should be actually cheaper per installation than their silver-graphite counterparts. Additional savings may be possible through elimination of provisions for spring loading and replacement of the brushes, since gold fibre brushes would be designed to outlast the machinery and to be simply loaded by fixing their position relative to the mating surface such that the fibres are suitably flexed.

Altogether, therefore, and assuming that the remaining developmental research is successful, the promise of gold fibre brushes and gold $Q M$ brushes for the future is very great. If it is realised, future high technology applications may be able to take advantage of electrical brushes which are virtually free of electrical noise, and which meet all reasonably expected performance requirements for low loss and high current density conduction. Moreover these brushes may well be cheaper than brushes now in use.

\section{References}

1 S.C. Rashleigh and R.A. Marshall, J. Appl. Pbys., 1978, 49, 2540-2542

$2 \mathrm{H}$. Kolm and P. Mongeau, IEEE Spectrum, 1982, 19, 30-36

3 C.M. Adkins and D. Kuhlmann-Wilsdorf, in 'Electrical Contacts-1979', Proc. Holm Conf. Electr. Contacts, Ill Inst. Technol., Chicago, IL., 1979, pp. $165-170$

4 See (3), pp. 171-184

5 C.M. Adkins and D. Kuhlmann-Wilsdorf, in 'Electrical Contacts - 1980', Proc. Holm Conf. Electr. Contacts, Ill. Inst. Technol., Chicago, IL., 1980, pp. 67.72

6 P. Reichner, in 'Electrical Contacts - 1979', Proc. Holm Conf. Electr. Contacts, Ill. Inst. Technol., Chicago, IL., 1979, pp. 191-197

7 P. Reichner, in 'Electrical Contacts - 1980', Proc. Holm Conf. Electr. Contacts, Ill. Inst. Technol., Chicago, IL., 1980, pp. 73-76

8 P.B. Haney, D. Kuhlmann-Wilsdorf and H.G.F. Wilsdorf, Wear, 1981 $73,261-282$

9 R. Holm, 'Electric Contacts', Springer Verlag, Berlin/Heidelberg/New York, 1967

10 H. Hertz, J. f. d. reine $u$. angew. Math., 1881, 92, 156, See also collected works

11 M.T. Huber, Ann. Phys, , 1904, 14, 153-163

12 S. Dillich and D. Kuhlmann-Wilsdorf, in 'Electrical Contacts - 1979', Proc. Holm Conf. Electr. Contacts, Ill. Inst. Technol., Chicago, II., 1979, pp. $185-190$

13 S. Dillich and D. Kuhlmann-Wilsdorf, in 'Electrical Contacts - 1982', Proc. Holm Conf. Electr. Contacts, Ill. Inst. 'Technol., Chicago, II., 1982, pp. 201-212
14 I R McNab, Wear, 1980,59,259-276

15 D. Kuhlmann-Wilsdorf, C.M. Adkins and H.G.F. Wilsdorf, U.S. Pat. Appl. Ser. No. 138, 716, filed April 9, 1980

16 Courtesy of AMP Inc., Harrisburg, PA . proprietary process

17 V. Srikrishnan, S. Dillich and D. Kuhlmann-Wilsdorf, in 'Electrical Contacts - 1978', Proc. Holm. Conf. Electr. Contacts, Ill. Inst. Technol. Chicago, IL., 1978, pp. 635-640

18 C.M. Adkins and D. Kuhlmann-Wilsdorf, Wear, 1981, 71, 119-125

19 D. Kuhlmann-Wilsdorf, in 'Fundamentals of Friction and Wear of Materials', in Proc. ASM Materials Science Seminar, edited by D. A. Rigney, Am. Soc. Met., Metals Park, OH., 1980, pp. 119-186

20 D. Kuhlmann-Wilsdorf, U.S. Pat. Appl. Ser. No. 156, 630, filed June 5 1980 